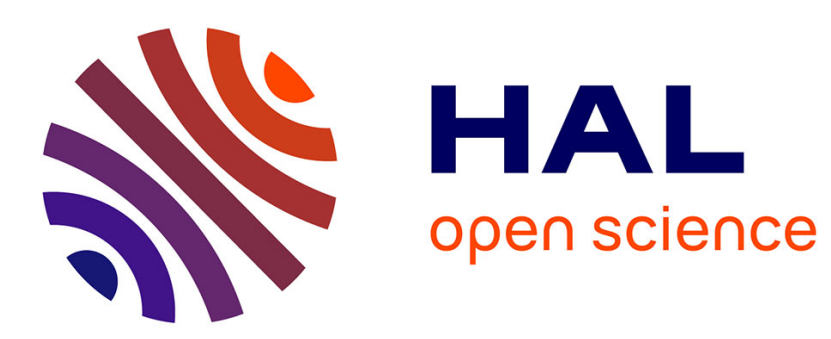

\title{
Perception et imitation du mouvement dans l'autisme: une question de temps
}

France Lainé, Carole Tardif, Stéphane Rauzy, Bruno Gepner

\section{To cite this version:}

France Lainé, Carole Tardif, Stéphane Rauzy, Bruno Gepner. Perception et imitation du mouvement dans l'autisme: une question de temps. Enfance, 2008, 2 (60), pp.140-157. hal-00354506

\section{HAL Id: hal-00354506 https://hal.science/hal-00354506}

Submitted on 20 Jan 2009

HAL is a multi-disciplinary open access archive for the deposit and dissemination of scientific research documents, whether they are published or not. The documents may come from teaching and research institutions in France or abroad, or from public or private research centers.
L'archive ouverte pluridisciplinaire HAL, est destinée au dépôt et à la diffusion de documents scientifiques de niveau recherche, publiés ou non, émanant des établissements d'enseignement et de recherche français ou étrangers, des laboratoires publics ou privés. 


\title{
Perception et imitation du mouvement dans l'autisme : une question de temps
}

\author{
France Lainé ${ }^{1}$, Carole Tardif ${ }^{1}$, \\ Stéphane Rauzy ${ }^{2}$, Bruno Gepner ${ }^{2,3}$
}

${ }^{1}$ PsyCLE, Centre de Psychologie de la Connaissance, du Langage et de l'Emotion, EA 3273, Université de Provence, UFR de Psychologie, 29 av. R. Schuman, 13621 Aix en Provence Cedex 1. france.laine@univ-provence.fr

${ }^{2}$ Laboratoire Parole et Langage, UMR CNRS 6057, Aix-en-Provence

${ }^{3}$ Centre Hospitalier Montperrin, Aix-en-Provence

\section{Mots-clés :}

Autisme ; perception du mouvement ; imitation

\section{Résumé :}

Un nombre croissant d'études a montré que certaines personnes autistes souffrent de troubles de la perception des mouvements biologiques pouvant affecter leur communication verbale et non verbale et leurs interactions sociales. Les mouvements de l'environnement iraient trop vite pour elles et déborderaient leurs capacités de traitement perceptif. Confirmant cette hypothèse explicative du trouble social dans l'autisme, des études antérieures ont montré que la reconnaissance d'expressions faciales est améliorée chez des enfants autistes lorsque ces expressions sont présentées de manière ralentie. Dans cette même perspective, notre présente étude vise à explorer, chez des enfants et adolescents autistes comparés à des enfants témoins normaux, l'impact de différentes vitesses de présentation de gestes corporels ou faciaux sur leur capacité à les imiter sur demande. Ces gestes, effectués par une personne, sont présentés sur ordinateur, en vitesse normale ou ralentie, grâce à un logiciel spécialement conçu à cet effet. Les résultats montrent d'une part que les enfants autistes, essentiellement ceux ayant un autisme plus sévère ou un âge de compréhension verbale plus bas, imitent moins bien les gestes que les enfants témoins, et, d'autre part, que leurs performances imitatives s'améliorent lorsque les gestes sont présentés lentement. Ces résultats pourraient ouvrir la voie à de nouvelles modalités de rééducation perceptive et communicative chez les personnes autistes.

\section{Title:}

Perception and Imitation of Biological Motion in Autism: a Matter of Time

\section{Keywords:}

Autism; motion perception; imitation

\section{Summary:}

An increasing amount of studies demonstrated the existence of biological motion integration deficit in individuals with autism, especially when velocity of movement is high. This type of movements (such as facial and body gestures) is intrinsically involved in social interactions: a rapid visual-motion processing deficit could therefore account for their verbal and non verbal comprehension and social interaction disorders. Previous studies showed that the speed of presentation of facial expressions to children with autism may influence their performance in recognition of these facial expressions, i.e., a slowed down presentation enhances their performance, particularly in those having the more severe autistic disorders. In the present study, we aimed at extending these findings to the field of imitation, i.e., to assess the influence of the speed of presentation of various gestures on the imitative abilities of children 
and adolescents with autism and normal control subjects. These gestures are meaningless, presented on a computer screen at normal and slowed down speeds by the means of a software especially dedicated to this research. Results show that autistic individuals, especially the more affected ones and those having the lower verbal comprehension level, imitate more poorly than their controls. More importantly, slow dynamic presentations enhance the performance in imitation of the autistic children. These findings may open new lines of interventions in the domain of perceptive and communicative reeducation in autistic individuals.

\section{Introduction}

Parmi les signes qui constituent le tableau clinique de l'autisme (APA, 2000; WHO, 1994), les altérations de la communication et les anomalies des interactions sociales représentent le déficit majeur du syndrome. Ce trouble social est présent chez toutes les personnes autistes, quels que soient leur âge, leur niveau de développement et de fonctionnement, et le degré de sévérité de leur autisme. Aussi, plusieurs modèles tentent d'expliquer ce trouble social en pointant différentes anomalies portant sur des fonctions essentielles aux interactions communicatives et sociales, qu'il s'agisse notamment de la lecture du regard (Baron-Cohen, Campbell, Karmiloff-Smith, \& Grant, 1995), de la lecture des émotions (Hobson, Ouston, \& Lee, 1988), de la théorie de l'esprit (Baron-Cohen, 2000), de la cohérence centrale (Frith, 1989), des fonctions exécutives (Russell, 1997) ou encore de l'imitation (Rogers \& Pennington, 1991).

Outre ces modèles, certains auteurs pensent que ce trouble social pourrait être lié à un désordre de la perception visuelle des mouvements (Gepner, 2001 ; Gepner, Lainé \& Tardif, 2005 ; pour une revue, voir Milne, Swettenham, \& Campbell, 2005). Telle est, dans cette présente étude, notre hypothèse de travail. Si la perception et l'intégration du mouvement fournissent des informations cruciales pour le développement du contrôle postural, de la coordination visuo-manuelle, de l'imitation, de l'attention conjointe et de la lecture des visages, alors une anomalie précoce de la perception du mouvement créerait autant de véritables obstacles qui s'installeraient entre le sujet et son environnement dès qu'il lui faudrait accéder à la compréhension de la parole et au décodage des expressions émotionnelles. D'ailleurs, des études ont révélé des similitudes dans le développement des enfants autistes et des enfants nés aveugles ou déficients visuels, concernant le délai d'apparition du langage et de la marche et les déficits sociaux (Greenspan \& Porges, 1984 ; Erin, 1990), soit autant de manifestations comportementales qualifiées dans l'autisme de signes indirects de déficience visuelle, tandis que l'absence ou les déficits d'attention conjointe et d'imitation visuelle lors des interactions sociales sont autant de signes directs de déficience visuelle (Mottron \& Belleville, 1998). Des différentes hypothèses perceptives du trouble social dans l'autisme, il ressort, pour certains, que cette difficulté à percevoir des stimuli mobiles s'expliquerait mieux par une capacité réduite à intégrer des informations perceptives complexes plutôt que par une incapacité à traiter efficacement les informations dynamiques en tant que telles (Bertone, Mottron, Jelenic, \& Faubert, 2003). Cette hypothèse est soutenue aussi par Williams, Goldstein, et Minshew (2006), pour lesquels le traitement des informations est altéré dans son ensemble, cette altération étant encore plus évidente lorsque la tâche à résoudre est complexe. Ces mêmes auteurs observent aussi une sorte de phénomène de compensation avec l'âge car les enfants autistes auraient des difficultés dans le traitement d'informations simples et complexes alors que seul le traitement d'informations complexes serait affecté chez les adultes. Pour d'autres auteurs, la difficulté à traiter les mouvements biologiques viendrait plus d'une difficulté à comprendre la signification sociale de ces mouvements humains en contexte social (Klin, Jones, Schultz, et Volkmar, 2003). 
Depuis une dizaine d'années, plusieurs études ont montré que les personnes autistes ont une gêne à détecter et à traiter les mouvements, qu'il s'agisse de mouvements biologiques (Gepner, Deruelle, \& Grynfeltt, 2001 ; Blake, Turner, Smoski, Pozdol, \& Stone, 2003) ou physiques (Gepner, Mestre, Masson, \& de Schonen, 1995 ; Spencer, O'Brien, Riggs, Braddick, Atkinson, \& Wattam-Bell, 2000 ; Milne, Swettenham, Hanse, Campbell, Jeffries, \& Plaisted, 2002), et ceci d'autant plus que les mouvements sont rapides (Gepner \& Mestre, 2002a) et/ou complexes (Spencer, et al., 2000 ; Milne et al., 2002 ; Bertone, Mottron, Jelenic, \& Faubert, 2003 ; Takarae, Minshew, Luna, Krisky, \& Sweeney, 2004). Tous ces résultats mettent en avant l'existence dans l'autisme d'une sensibilité réduite aux mouvements (Milne et al., 2005, pour une revue) et d'un déficit de l'intégration d'informations perceptives dynamiques et complexes. Des travaux en imagerie cérébrale viennent confirmer ces données en mettant en évidence des dysfonctionnements au niveau du sillon temporal supérieur chez les personnes autistes (Cody, Pelphrey, \& Piven, 2002), région normalement impliquée dans le traitement visuel des mouvements biologiques (mouvements du corps, des mains, des lèvres et des yeux, voir Allison, Puce, \& McCarthy, 2000, pour une revue).

Certains travaux suggèrent donc que les désordres de la perception des mouvements observés dans l'autisme sont en particulier liés à leur vitesse (Gepner \& Mestre, 2002a, 2002b; Gepner \& Tardif, 2006). L'existence de troubles précoces du traitement visuel du mouvement chez certaines personnes autistes pourrait expliquer plusieurs de leurs signes cliniques, leurs conséquences invalidantes et les mécanismes compensatoires adaptatifs mis en place (Gepner, 2006). Les personnes avec autisme ne seraient pas aveugles aux mouvements mais présenteraient une hypo- ou une hypersensibilité aux mouvements, qui seraient d'autant plus manifestes que les mouvements sont rapides. Ces derniers seraient alors négligés et non perçus, ou perçus comme des stimuli aversifs, provoquant par conséquent un évitement du regard et un manque d'intérêt porté aux visages humains, ce que Kanner (1943) avait déjà noté comme étant typique de ces enfants, ce qu'évoque aussi Grandin (1997) («Il se pourrait que les problèmes de contact oculaire rencontrés par les autistes résultent en partie d'une incapacité à supporter le mouvement des yeux d'un interlocuteur »). Or, dans la vie de tous les jours, visage et regard sont constamment impliqués dans les interactions sociales lors desquelles ils sont mobiles et très vite fluctuants. Cet évitement du regard ou ce manque d'intérêt pour les visages, liés au fait que les mouvements faciaux sont trop rapides, expliquerait en partie leurs troubles du traitement des visages (Gepner, de Gelder, \& de Schonen, 1996). Gepner (2001) propose en conséquence de ralentir les mouvements impliqués dans les interactions sociales (expressions faciales, gestes, postures des partenaires d'interaction) afin qu'ils puissent être mieux traités, reconnus, assimilés, et, à terme, compris. Cette hypothèse a déjà été testée auprès d'enfants autistes afin d'évaluer leur capacité à reconnaître des expressions faciales présentées en vitesse normale et ralentie (Lainé, Tardif, \& Gepner, sous presse ; Tardif, Lainé, Rodriguez, \& Gepner, 2007). Les résultats ont révélé, d'une part, que les enfants autistes reconnaissent mieux ces expressions lorsqu'elles sont présentées de manière ralentie, et, d'autre part, que ce sont les enfants avec un autisme plus sévère qui bénéficient le plus de cette présentation ralentie. Ces résultats confirment celles de Blake et al. (2003) et de Gepner et Mestre (2002a) concernant l'existence d'un lien entre le degré de sévérité d'autisme et l'importance du trouble de la perception des mouvements. Dans ces mêmes études, Lainé et al. (sous presse) et Tardif et al. (2007) ont également mis en évidence que globalement les enfants autistes imitent spontanément (sans demande explicite de la part de l'expérimentateur) les expressions présentées sur ordinateur (ce qui est peu ou pas observé chez les témoins), et qu'ils les imitent d'autant plus qu'elles sont présentées de manière ralentie.

Dans la suite de ces travaux, nous souhaitons à présent examiner chez des enfants et adolescents autistes l'effet du ralentissement de différents mouvements biologiques sur leurs 
capacités à les imiter sur demande. En effet, l'imitation est aussi une composante essentielle des interactions sociales faisant partie des premiers outils de communication à disposition du jeune enfant (Nadel, 2006). Aussi, un trouble de l'imitation pourrait avoir des conséquences tant au niveau des apprentissages sensori-moteurs dès le plus jeune âge, qu'au niveau des habiletés à échanger avec autrui et à comprendre ses intentions. D'ailleurs, des difficultés à imiter ont été décrites pour la première fois chez des enfants autistes par de Meyer et al. (1972). Depuis, des travaux suggèrent qu'un déficit imitatif existe chez certains enfants autistes et pourrait même représenter un des signes précoces du syndrome (Heimann \& Ullstadius, 1996), se manifestant plutôt comme un retard que comme une atypicalité développementale (Williams, Whiten, \& Singh, 2004). D'autres travaux encore envisagent que l'imitation puisse être un déficit central de l'autisme, dont l'origine se situerait au niveau d'un trouble de la formation et de la coordination des représentations spécifiques soi-autrui (Rogers \& Pennington, 1991), ou, plus généralement, d'un trouble du traitement des informations complexes requis par les stimulations sociales (Smith \& Bryson, 1994). Ce déficit primaire se manifesterait en premier lieu par un trouble de l'imitation accompagné d'une cascade de perturbations venant entraver l'ensemble du développement affectif, social, communicationnel et cognitif de l'enfant. Si ces auteurs avancent l'idée que ce déficit imitatif est présent chez tous les enfants autistes, d'autres en nuancent l'universalité, telles que Nadel et Aouka (2006) qui mettent en évidence, à l'aide d'une épreuve d'imitation de gestes sans signification (épreuve empruntée à l'étude neurologique de l'apraxie, Goldenberg, 1996, 1999), que des enfants autistes de niveau cognitif élevé sont de bons imitateurs, alors que ceux de niveau cognitif plus bas échouent massivement cette épreuve. Elles concluent alors que le trouble praxique n'est pas une caractéristique universelle de l'autisme.

Au-delà de ce débat autour de l'imitation plus ou moins déficitaire ou plus ou moins préservée dans l'autisme, nous cherchons dans cette étude à savoir si les performances imitatives des personnes autistes pourraient être améliorées par une présentation ralentie des mouvements biologiques à imiter.

\section{Problématique et objectif de la recherche}

A l'heure actuelle, une littérature croissante fait état de l'existence de troubles de perception des mouvements biologiques chez les personnes atteintes d'autisme. Ces troubles pourraient expliquer les déficits imitatifs rencontrés dans cette population : un mouvement mal perçu sera a fortiori mal imité, voire non imité. Nous faisons l'hypothèse que ces stimuli dynamiques seraient en fait trop rapides pour être perçus correctement par les personnes autistes, hypothèse déjà mise à l'épreuve et confirmée dans des études antérieures sur la reconnaissance d'expressions faciales dynamiques (Lainé et al., sous presse ; Tardif et al., 2007). Nous supposons qu'un ralentissement des mouvements à imiter, réalisé à l'aide d'un logiciel informatique, augmenterait la production d'imitations chez des enfants et adolescents autistes. Si cette hypothèse se vérifiait, une telle technique de ralentissement pourrait alors représenter une ressource importante dans les dispositifs d'aide à l'apprentissage et à la communication pour les personnes autistes.

L'objectif de cette recherche est donc d'étudier l'impact d'une présentation ralentie de mouvements corporels, manuels et faciaux présentés à des enfants et adolescents autistes, et à des enfants témoins au développement typique, sur leurs performances imitatives.

\section{Méthodologie de la recherche \\ 3.1. Population}

Le groupe expérimental est constitué de 14 enfants et adolescents autistes ( 2 filles et 12 garçons), âgés de 6 ans 8 mois à 17 ans 10 mois $(\mathrm{M}=10 ; 11$ ans ; E.T. $=3 ; 6$ ans $)$, diagnostiqués selon les critères du DSM-IV-TR (APA, 2000) et pris en charge dans des 
établissements hospitaliers et scolaires d'Aix et Marseille (hôpital de jour, SESSAD et classes spécialisées). Leur degré de sévérité d'autisme évalué à l'aide de la CARS (Childhood Autism Rating Scale ; Schopler, Reichler, de Vellis, \& Daly, 1980) révèlent des scores compris entre 31 et 38. Afin de pallier l'hétérogénéité de cette population et de pouvoir affiner nos résultats, nous avons constitué deux sous-groupes plus homogènes sur la base de leur proximité de score à la CARS, à savoir, un sous-groupe de 6 enfants et adolescents autistes ayant un score supérieur ou égal à 36,5, que nous appelons « autisme modéré-sévère » (au regard de notre échantillon total), et un sous-groupe de 8 ayant un score inférieur ou égal à 36 que nous appelons « autisme léger-modéré » (tableau 1).

Leur âge de compréhension du vocabulaire (ACV) est mesuré au moyen de l'Echelle de Vocabulaire en Image Peabody (EVIP, Dunn, Thériault-Whalen, \& Dunn, 1993). Il permet d'évaluer le niveau de réception du vocabulaire de l'enfant par désignation d'images. Cet âge nous permet de vérifier que les enfants autistes comprennent la consigne verbale donnée pour effectuer la tâche, et nous sert également de base d'appariement avec les enfants tout-venant du groupe témoin. Leur niveau en communication expressive ne fait pas ici l'objet d'une évaluation puisque la tâche, pour être effectuée, ne requiert pas l'utilisation du langage.

Le groupe témoin est constitué de 14 enfants tout-venant appariés aux enfants et adolescents autistes sur la base de l'ACV et du sexe (Tableau 1). Ils sont âgés de 1 an 10 mois à 7 ans 5 mois $(\mathrm{M}=3 ; 10$ ans ; E.T. $=1 ; 8$ ans) ; ceux ayant obtenu des ACV trop élevés ou trop faibles par rapport à la moyenne de leur groupe d'âge ont été exclus de l'échantillon.

Tableau 1 : Présentation de la population

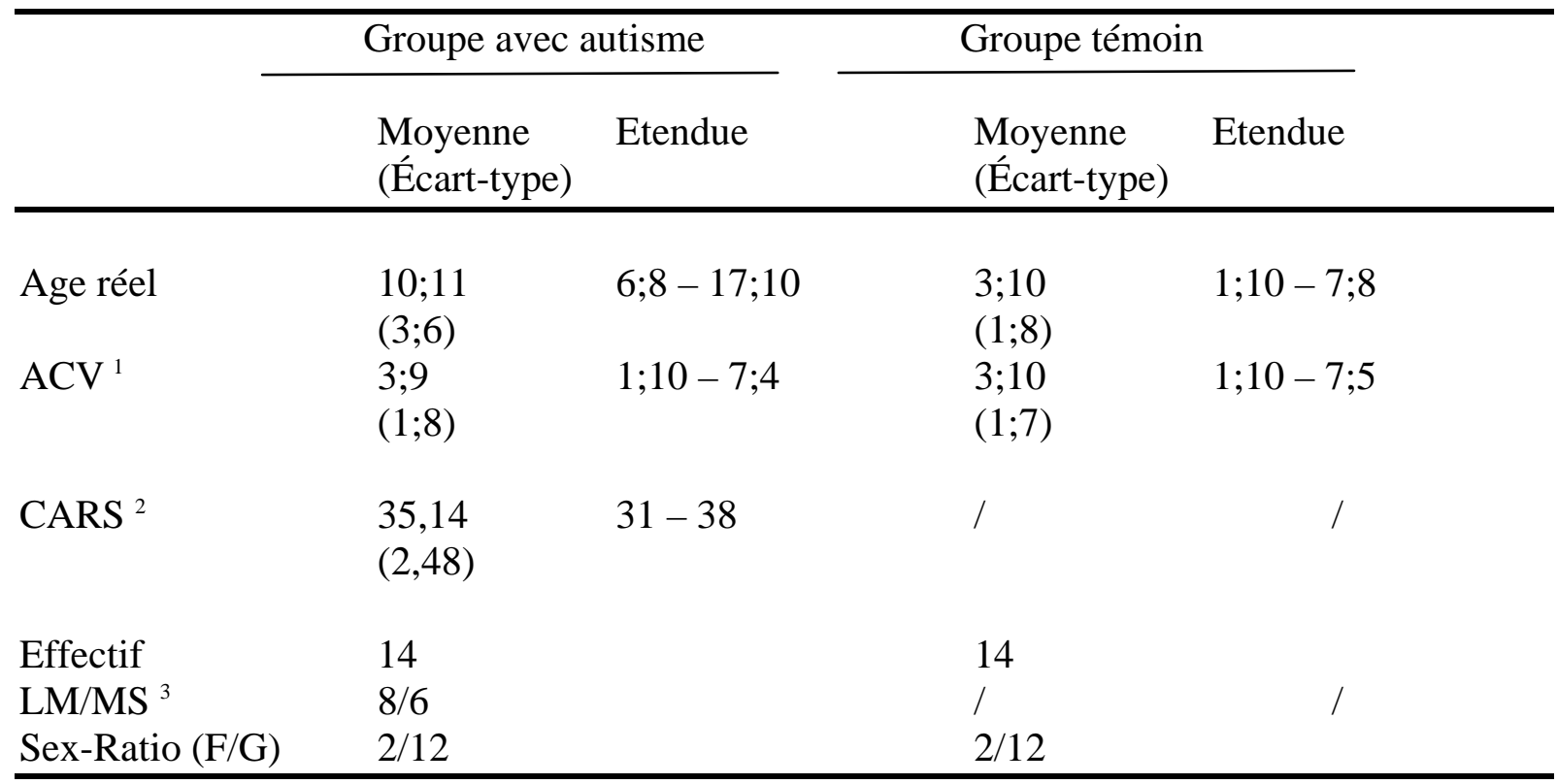

Légende :

${ }^{1}$ ACV : âge de compréhension du vocabulaire, mesuré au moyen de l'EVIP.

${ }^{2}$ CARS : score indiquant le degré de sévérité d'autisme.

${ }^{3}$ LM/MS : autisme Léger-Modéré ; autisme Modéré-Sévère

\subsection{Matériel}

Afin de pouvoir présenter aux participants différents gestes à imiter dans plusieurs vitesses, nous avons conçu et développé un logiciel informatique permettant de ralentir les 
mouvements sans en dénaturer la fluidité. Le système offre la possibilité de ralentir de façon synchrone la vitesse d'un signal composé d'une entrée vidéo (par exemple la sortie d'une caméra vidéo filmant un interlocuteur) et d'une entrée audio associée à l'entrée vidéo (le message oralisé par l'interlocuteur). Le ralentissement du signal audio sans altérer la hauteur des sons produits est réalisé par adaptation d'un algorithme de type SOLA (Synchronized OverLap Add algorithm) qui permet de ralentir la vitesse d'une séquence audio enregistrée (une voix par exemple) jusqu'à un facteur 3 environ sans perte d'intelligibilité. La synchronisation du ralentissement audio et du ralentissement du signal vidéo est réalisée dans notre prototype via un contrôle événementiel avec une précision temporelle de 20 millisecondes. Une interface graphique permettant de présenter les planches de stimuli au sujet vient compléter le dispositif. Chaque planche est définie par un fichier de paramètres incluant notamment le nombre et le positionnement des stimuli sur la planche, la nature des stimuli (image fixe, séquence vidéo, séquence audio ou séquence audio-vidéo), la vitesse de ralentissement, etc... Pour la présente étude, les stimuli se limitent à la présentation de séquences vidéo (sans signal audio associé) enregistré à l'aide d'une caméra vidéo. Le logiciel a été installé sur un ordinateur portable (DELL® Latitude D600).

\subsection{Protocole}

L'enfant voit sur l'écran d'un ordinateur portable une femme faire un geste qu'il doit ensuite reproduire. Celle-ci réalise 20 gestes au total : 5 concernant le visage (par exemple, gonfler les joues, fermer les yeux), 5 concernant les mains (par exemple, fermer le poing), 4 concernant le corps (par exemple, sauter deux fois) et 6 concernant les bras (par exemple, faire le moulin avec les deux bras) [voir annexe pour une description plus détaillée de chaque geste]. Ces gestes sont issus de batteries d'épreuves étalonnées (K-ABC, Kaufman \& Kaufman, 1993 ; PEP-R, Schopler \& Reichler, 1979 ; Gesture Test, Cermak, Coster, \& Drake, 1980) ou ont déjà été testés dans d'autres études (Beadle-Brown \& Whiten, 2004 ; Green, Baird, Barnett, Henderson, Huber, \& Henderson, 2002).

Chaque geste est présenté dans 3 vitesses différentes : 1) une vitesse dite normale, de la vie quotidienne, notée $\mathrm{V} 1 ; 2)$ une vitesse dite lente, obtenue grâce à un ralentissement par deux de la vitesse normale, notée $\mathrm{V} 2$; et 3 ) une vitesse dite très lente, obtenue grâce à un ralentissement par deux et demi de la vitesse normale, notée V3. Les deux vitesses ralenties le sont au moyen du logiciel. Le choix de ces deux vitesses répond à une première exploration $\mathrm{du}$ ralentissement possible pour en tester l'impact sur les performances à imiter des participants. Par la suite, d'autres essais seront à faire avec divers degrés de ralentissement afin de trouver le ralenti optimal pour chaque enfant. En moyenne, les séquences durent 2 secondes en V1, 4 secondes en V2 et 5 secondes en V3. Nous obtenons ainsi 20 gestes à imiter, présentés dans les 3 vitesses dans un ordre aléatoire, donnant un total de 60 items.

Les résultats de chaque enfant sont évalués en fonction de la qualité de reproduction du geste. Si l'enfant imite le geste dans son intégralité, il obtient un score de 2 points; si l'imitation est partielle (par exemple, l'enfant ne saute qu'une fois alors qu'il doit sauter deux fois), il obtient un score de 1 point ; enfin, s'il réalise un autre mouvement ou ne fait rien, son score est nul.

\subsection{Procédure}

Nous rencontrons l'enfant sur son lieu d'accueil (hôpital de jour, classe) ou à son domicile selon le contexte dans lequel il est le plus à l'aise pour participer à l'expérience. Dans tous les cas, nous testons l'enfant dans une pièce au calme. Une personne adulte familière peut rester avec lui pendant la passation s'il a besoin d'être rassuré.

Nous commençons d'abord par évaluer l'âge de compréhension de vocabulaire avec l'EVIP, puis nous faisons passer l'expérience sur ordinateur. 
L'enfant voit à l'écran une femme (toujours la même) faire un mouvement, après quoi un écran noir apparaît. Nous demandons alors à l'enfant de reproduire ce qu'il vient de voir à l'écran. L'écran noir nous permet de nous assurer que l'enfant ne va pas reproduire le geste uniquement en fonction de la dernière image qu'il a sous les yeux mais bien en fonction de l'ensemble du geste en mouvement.

La consigne de cette expérience est donnée comme suit : «Tu vois la dame à l'écran faire un geste. A la fin de son geste, tu devras refaire exactement comme elle ». Avant de débuter l'expérience proprement dite, nous proposons à l'enfant une phase de démonstration comprenant 6 items : pour chacun des 6 items, l'expérimentateur réalise la consigne en reproduisant le geste présenté, fournissant ainsi un exemple à l'enfant. Dès que la phase de démonstration est terminée, l'expérience démarre. Nous faisons autant de pauses que nécessaire pour l'enfant.

\subsection{Hypothèses}

Nous faisons l'hypothèse:

- que le groupe avec autisme obtiendra globalement de moins bonnes performances en imitation que le groupe témoin ;

- que dans le groupe avec autisme, les performances imitatives seront meilleures lorsque les items sont présentés en vitesses ralenties (V2 et V3), comparativement à la vitesse normale (V1);

- que les enfants du sous-groupe avec autisme modéré-sévère (MS) seront davantage aidés par les présentations ralenties (V2 et V3) que les enfants du sous-groupe avec autisme légermodéré (LM).

\section{Résultats}

Nous avons procédé à une analyse de la variance à mesure répétée (ANOVA) sur les résultats en imitation des deux groupes, selon le schéma suivant : 2 (Groupe) x 3 (Vitesse de présentation), avec le score à la tâche comme variable dépendante, et la vitesse de présentation comme mesure répétée.

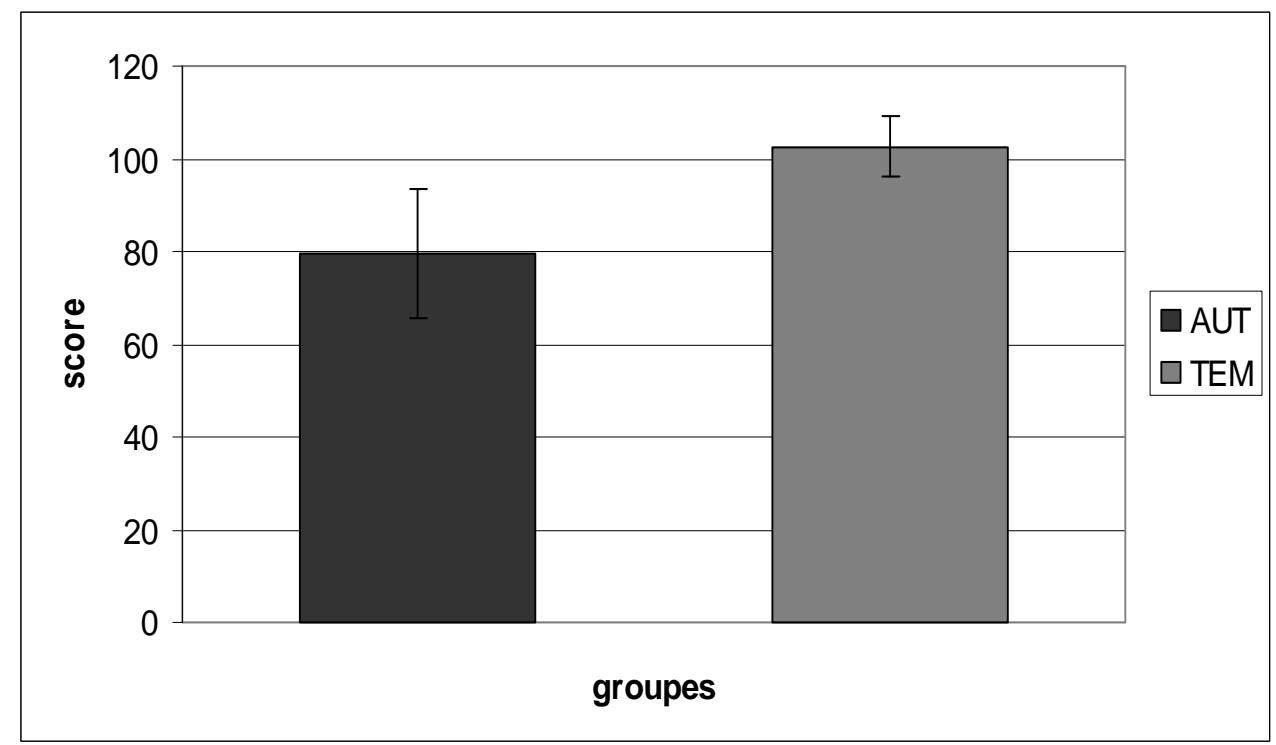

Figure 1 : Score moyen d'imitations pour le groupe avec autisme (AUT) et pour le groupe témoin (TEM) 


\subsection{Comparaisons intergroupes}

L'analyse de variance à mesure répétée révèle que le groupe avec autisme obtient un score total en imitation inférieur à celui du groupe témoin $[\mathrm{F}(1,26)=6,68$ et $\mathrm{p}=.02]$ (Figure $1)$. D'autre part, nous ne pouvons noter d'effet principal de la condition « vitesse » $[\mathrm{F}(2,52)$ $=1,45$ et $\mathrm{p}=.24]$, ni d'effet d'interaction « groupe $\mathrm{x}$ vitesse $»[\mathrm{~F}(2,52)=2,82$ et $\mathrm{p}=.19]$.

L'ANOVA révèle un effet simple du groupe $[\mathrm{F}(3,24)=7,35$ et $\mathrm{p}=.001]$, quelle que soit la vitesse de présentation des gestes à imiter [comparaisons planifiées en $\mathrm{V} 1: \mathrm{F}(1,26)=$ $6,41$ et $\mathrm{p}=.02$; en $\mathrm{V} 2: \mathrm{F}(1,26)=5,63$ et $\mathrm{p}=.02$; en $\mathrm{V} 3: \mathrm{F}(1,26)=7,28$ et $\mathrm{p}=.01]$. Les analyses post hoc (réalisées à l'aide du HSD de Tukey) indiquent plus précisément que le score total en imitation du groupe avec autisme léger-modéré et du groupe témoin sont comparables $(\mathrm{p}=.63)$, alors que celui du groupe avec autisme modéré-sévère est inférieur à la fois à celui du groupe témoin $(\mathrm{p}=.0009)$ et du groupe avec autisme léger-modéré $(\mathrm{p}=.01)$. Par conséquent, la différence observée entre le score total du groupe avec autisme et celui du groupe témoin est imputable au groupe avec autisme de niveau modéré-sévère.

\subsection{Analyses des performances au sein du groupe avec autisme}

Un calcul de corrélation à l'aide du Tau de Kendall met en évidence une relation négative entre le score total en imitation et le score à la CARS, signifiant une évolution inverse de ces deux variables $(\tau=-.44, \mathrm{p}=.03)$.

Il existe également un lien entre le score total en imitation et l'âge de compréhension verbale, révélant que les deux évoluent parallèlement et dans le même sens $(\tau=.41, p=.04)$.

Nous avons également effectué des comparaisons planifiées en tant qu'analyses $a$ priori afin d'étudier spécifiquement l'effet de la vitesse dans le groupe avec autisme.

Celles-ci révèlent une amélioration des performances imitatives en vitesse lente comparativement à la vitesse normale $[\mathrm{F}(1,26)=4,4$ et $\mathrm{p}=.04]$. Ce résultat significatif n'est pas retrouvé pour le groupe témoin $[\mathrm{F}(1,26)=0,06$ et $\mathrm{p}=.81]$. En revanche, les performances obtenues en vitesse très lente ne diffèrent pas de celles obtenues en vitesse normale $[\mathrm{F}(1,26)=1,42$ et $\mathrm{p}=.24]$ (Figure 2$)$.

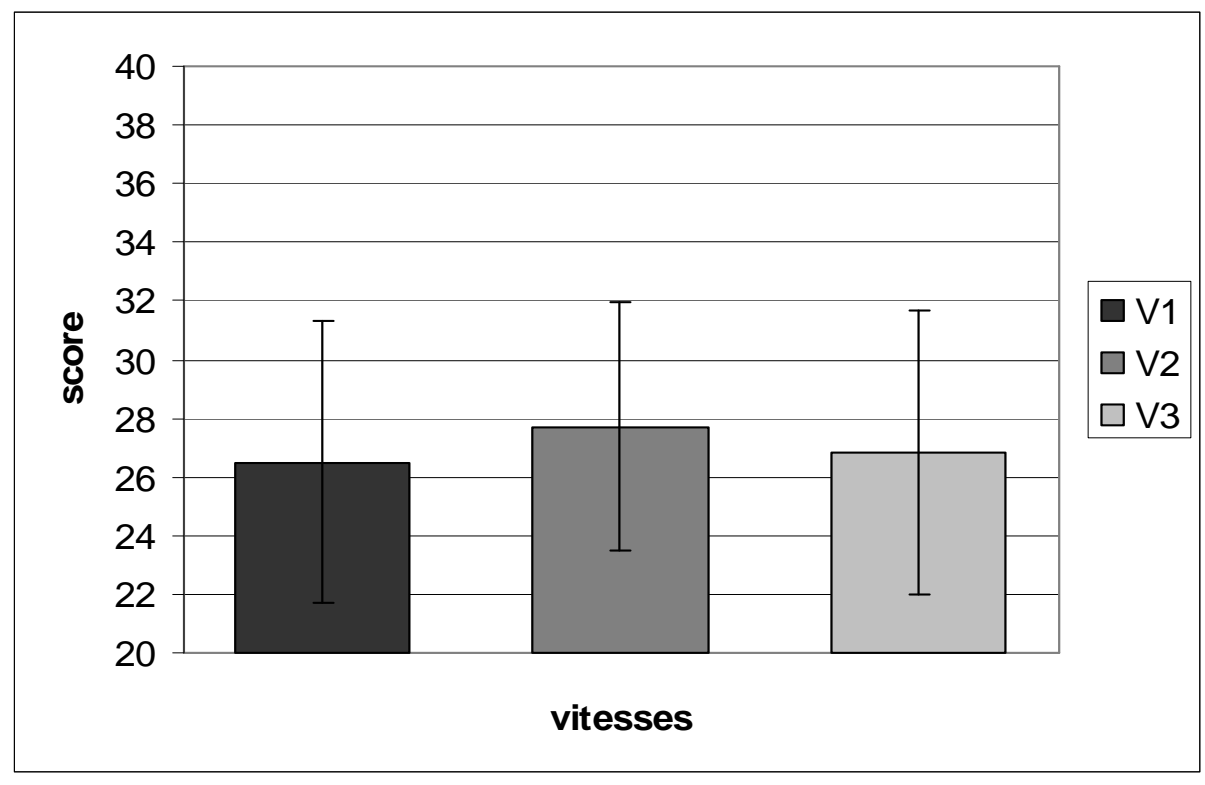

Figure 2 : Score moyen d'imitations en fonction de la vitesse pour le groupe avec autisme 
Nous avons également souhaité explorer la question du lien entre le degré de sévérité d'autisme et l'intensité du trouble perceptif de la vision des mouvements. Nous avons donc évalué le gain apporté par les vitesses ralenties dans les deux sous-groupes avec autisme, en considérant que les enfants autistes étaient aidés par les vitesses ralenties dès lors qu'ils obtenaient au moins $10 \%$ de plus à leur score, dans au moins une des deux vitesses ralenties (V2 et/ou V3), comparativement à leur score en vitesse normale (V1).

Tableau 2 : Nombre d'enfants autistes aidés par le ralenti pour imiter des gestes

\begin{tabular}{llc}
\hline \multicolumn{1}{c}{ Enfants } & $\begin{array}{l}\text { Aidés par le } \\
\text { ralenti }\end{array}$ & $\begin{array}{l}\text { Non aidés par le } \\
\text { ralenti }\end{array}$ \\
\hline $\begin{array}{l}\text { avec autisme léger-modéré } \\
(\mathrm{n}=8)\end{array}$ & 2 & 6 \\
$\begin{array}{l}\text { avec autisme modéré-sévère } \\
(\mathrm{n}=6)\end{array}$ & 5 & 1 \\
\hline
\end{tabular}

Un test exact de Fisher permet de mettre en évidence que seul le groupe avec autisme modéré-sévère bénéficie d'une présentation ralentie de manière significative $(p=.05)$ (Tableau 2). Il semble donc bien exister un lien entre le degré de sévérité d'autisme et le trouble perceptif de la vision des mouvements.

\section{Discussion et conclusion}

L'examen des données recueillies permet d'approfondir les résultats de la littérature concernant le déficit imitatif observé dans l'autisme. Globalement, le groupe avec autisme présente plus de difficultés à imiter sur demande des mouvements biologiques (faciaux, manuels et corporels) que le groupe témoin de même âge de compréhension verbale. Ce résultat suggère que le déficit imitatif observé dans l'autisme ne serait pas seulement le fait d'un retard développemental mais également d'une atypicalité développementale, puisque même pour les enfants autistes plus âgés, leurs années d'expérience ne comblent pas ce décalage. En effet, à titre d'exemple, un enfant autiste de 8 ans d'âge réel et de 1 an 10 mois d'âge de compréhension du vocabulaire est apparié à un enfant témoin de 1 an 10 mois. De ce fait, quand nous comparons ces deux enfants, nous prenons en compte leurs expériences respectives, celle de l'enfant autiste dépassant largement celle de l'enfant témoin. Et pourtant les performances imitatives de l'enfant autiste sont plus faibles. C'est pourquoi ce résultat indiquerait plus une anomalie de l'imitation qu'un retard à imiter. Cette suggestion est renforcée par une autre étude de l'impact du ralenti sur l'imitation (Lainé, Tardif, \& Gepner, en préparation) comparant un groupe d'enfants autistes à des groupes d'enfants normaux et d'enfants retardés mentaux (atteints de trisomie 21), et dont les premiers résultats révèlent que les enfants autistes sont moins performants que les enfants retardés dans cette tâche, et qu'ils sont les seuls à être aidés par le ralenti. Par ailleurs, le CHAT (Checklist for Autism in Toddlers, questionnaire de dépistage précoce de l'autisme de Baron-Cohen et al., 2000) considère le trouble imitatif comme l'un des six domaines spécifiquement déficitaires de ce syndrome. Cependant, si les enfants autistes imitent moins et moins bien que les enfants toutvenant, aucun d'entre eux n'a présenté de déficit total d'imitation. Ils conservent donc des 
capacités qui ne sont pas en «tout ou rien », comme le montrent d'autres observations (Tardif \& Plumet, 2000 ; Beadle-Brown \& Whiten, 2004 ; Nadel, 2004).

$\mathrm{Au}$ sein du groupe avec autisme, la répartition en deux sous-groupes plus homogène nous a permis d'explorer la relation entre le déficit imitatif et le degré de sévérité d'autisme. Il s'avère alors que les enfants autistes d'atteinte moins sévère sont les plus compétents pour imiter. Ceci suggère par conséquent que les performances en imitation gestuelle varient selon le niveau de fonctionnement de l'enfant, notamment en terme de communication et imitation sociales, comme le soulignent également Nadel et Potier (2002). Ceci explique que les enfants avec autisme léger-modéré, ayant des capacités sociales plus élaborées, présentent des compétences imitatives comparables à celles d'enfants tout-venant de même niveau développemental, contrairement aux enfants avec autisme modéré-sévère qui présentent des compétences inférieures à celles observées chez les enfants tout-venant de niveau équivalent. Ces résultats confirment les travaux de Nadel (2006) et nuancent la thèse d'un déficit primaire d'imitation dans l'autisme de Rogers et Pennington (1991). Si les enfants autistes les plus sévèrement atteints imitent moins bien, c'est peut-être car ils ne comprennent pas l'enjeu de telles imitations, dans un contexte expérimental, mais également dans la vie de tous les jours. Ne comprenant pas l'enjeu social et communicatif des comportements imitatifs, ils imiteraient moins le partenaire d'interaction. C'est pourquoi cette compétence doit être travaillée, surtout avec les enfants les plus sévèrement atteints. A ce titre, plusieurs auteurs montrent que des sessions répétées d'imitation améliorent les comportements sociaux des enfants autistes (notamment non verbaux) et recommandent ce type d'intervention précoce pour améliorer leur fonctionnement social (Nadel, et al., 2000 ; Field, Field, Sanders, et Nadel, 2001 ; Escalona, Field, Nadel, \& Lundy, 2002).

Conformément à notre hypothèse de départ, le groupe avec autisme imite d'autant mieux les mouvements observés sur demande que ceux-ci sont ralentis. Ce résultat complète ainsi ceux de nos études antérieures ayant mis en évidence que certains enfants autistes imitent spontanément les mouvements faciaux présentés sur ordinateur, et ce d'autant plus qu'ils sont ralentis (Lainé et al., sous presse ; Tardif et al., 2007). Globalement, pour l'ensemble du groupe avec autisme, la vitesse lente semble optimale pour permettre l'imitation, même si une observation au cas par cas révèle que quelques enfants sont aidés préférentiellement par la vitesse très lente, démontrant l'existence d'un seuil optimal de ralentissement spécifique à chaque enfant. D'autre part, la présentation ralentie par deux et demi pourrait être trop lente pour certains enfants et entraîner chez eux des biais locaux favorisant un traitement local et pénalisant par conséquent une analyse plus globale du mouvement (Frith, 1989; Mottron \& Belleville, 2003 ; Tardif et al., 2007), alors que la présentation ralentie par deux uniquement l'avantagerait au contraire. Une autre interprétation possible serait que le ralenti par deux et demi génèrerait une baisse d'attention chez certains enfants autistes (Landry \& Bryson, 2004). Parallèlement, aucun effet du ralentissement des mouvements n'est observé chez les enfants tout-venant.

Par ailleurs, nous avons exploré la relation entre trouble perceptif de la vision des mouvements et sévérité du trouble autistique et constaté que le ralentissement aide davantage les enfants avec autisme de niveau modéré-sévère que ceux de niveau léger-modéré. Il y aurait donc un lien entre le degré de sévérité d'autisme et le trouble de la perception des mouvements, lien par ailleurs déjà mis en évidence dans d'autres études (Gepner \& Mestre, 2002a; Blake et al., 2003 ; Lainé et al., sous presse ; Tardif et al., 2007).

Notre étude permet de montrer que la procédure de ralentissement est susceptible d'aider les enfants autistes, notamment les plus sévèrement atteints, à extraire et à traiter les informations nécessaires pour imiter les mouvements présentés, et permet d'étayer la thèse d'une anomalie précoce de la perception et de l'intégration visuo-motrice du mouvement rapide dans l'autisme (Gepner, 2001, 2006; Gepner \& Mestre, 2002b). Cette anomalie pourrait 
être considérée comme un signe précurseur permettant, d'une part, de détecter précocement le trouble autistique chez certains enfants, et d'autre part, d'envisager à l'avenir une base simple et efficace pour l'éducation et la rééducation perceptive précoce d'au moins certains enfants autistes.

Cependant, il faut rester prudent quant à la spécificité des troubles de la vision des mouvements dans l'autisme. En effet, des études effectuées dans d'autres pathologies ont également révélé l'existence de tels troubles, notamment dans la dyslexie (Talcott, Hansen, Assoku, \& Stein, 2000 ; Hansen, Stein, Orde, Winter, \& Talcott, 2001), dans le syndrome de Williams (Atkinson, King, Braddick, Nokes, Anker, \& Braddick, 1997), dans le syndrome de l'X-fragile (Kogan, Boutet, Cornish, Zangenehpour, Mullen, \& Holden, 2004), ou encore chez des individus avec retard mental non spécifié (Fox, \& Oross, 1990 ; Sparrow, Shinkfield, Day, \& Zerman, 1999). Le fait qu'un trouble des apprentissages soit associé à ces différentes pathologies amènent Milne et al. (2005) à se poser la question de l'existence d'un lien entre le trouble de la vision des mouvements et un trouble d'apprentissage plus général. Mais ces auteurs rejettent cette hypothèse, car ce trouble perceptif visuel est également retrouvé chez des individus ayant un syndrome d'Asperger ou un autisme sans retard mental, et donc sans trouble des apprentissages associé (Milne et al., 2002 ; Bertone et al., 2003). Ce trouble serait donc lié à une dimension autre que le retard des acquisitions, dimension restant encore à éclairer.

En conclusion, faciliter les conduites imitatives par le ralentissement éco-biologique ou artificiel (via notre logiciel) des stimuli, pourrait favoriser les capacités de communication et d'interaction sociale des enfants atteints d'autisme (et peut-être d'autres pathologies). L'imitation est une activité cardinale du développement qui permet non seulement d'apprendre ce que l'on voit faire mais aussi d'attirer l'attention de l'autre et de montrer que l'on s'intéresse à la même chose. En cela, elle participe à la découverte de soi et de l'autre en tant que personne. Comme le souligne Nadel (2005), il n'est pas essentiel de comparer la quantité et le niveau des imitations des enfants avec ou sans autisme, «ce qui est primordial est d'exploiter ce qui existe en chaque enfant autiste $\gg$ (p. 355). Les perspectives offertes par notre approche pourraient à l'avenir présenter un intérêt majeur pour l'enfant autiste, sa famille et le travail des professionnels.

\section{Remerciements :}

Nous remercions le CNRS pour avoir contribué à financer cette recherche dans le cadre de l'appel à projet 2003 du programme interdisciplinaire «Cognition et Traitement de l'Information ».

Nous remercions la Fondation Orange (France Télécom) pour avoir contribué à financer cette recherche en allouant une bourse à France Lainé, dans le cadre de son doctorat, en 20052006.

Nous remercions Akselle Di Battista et Anne-Laurie Ndjikessi, étudiantes en Master 1 de Psychologie, pour leur contribution au recueil et à l'analyse des données.

Nous remercions Philippe Blache, directeur de recherche au CNRS (UMR 6057), pour sa collaboration scientifique à l'élaboration du logiciel de ralentissement.

\section{Références Bibliographiques}

Allison, T., Puce, A., \& McCarthy, G. (2000). Social perception from visual cues: Role of the STS region. Trends in Cognitive Sciences, 4, 267-278.

American Psychiatric Association, (2000). Diagnostic and Statistical Manual of Mental Disorders: Text Revision (DSM IV TR). Washington, D.C.: American Psychiatric Association. 
Atkinson, J., King, J., Braddick, O., Nokes, L., Anker, S., \& Braddick, F. (1997). A specific deficit of dorsal stream function in Williams syndrome. Neuroreport, 8, 1919-1922.

Baron-Cohen, S. (2000). Theory of mind and autism: a fifteen year review, in: S. BaronCohen, H. Tager-Flusberg, \& D. Cohen (eds), Understanding other minds: perspectives from developmental cognitive neuroscience $\left(2^{\text {nd }}\right.$ ed). Oxford: Oxford University Press.

Baron-Cohen, S., Campbell, R., Karmiloff-Smith, A., \& Grant, J. (1995). Are children with autism blind to the mentalistic significance of the eyes ? British Journal of Devevelopmental Psychology, 13, 379-398.

Baron-Cohen, S., Wheelwright, S., Cox, A., Baird, G., Charman, T., Swettenham, J., Drew, A., \& Doehring, P. (2000). Early Identification of Autism by the CHeklist for Autism in Toddlers (CHAT). Journal of the Royal Society of Medicine, 93, 521-525.

Beadle-Brown, J.D., \& Whiten, A. (2004). Elicited imitation in children and adults with autism: Is there a deficit? Journal of Intellectual \& Developmental Disability, 29, $147-$ 163.

Bertone, A., Mottron, L., Jelenic, P., \& Faubert, J. (2003). Motion perception in autism: A "Complex" Issue. Journal of Cognitive Neuroscience, 15, 1-8.

Blake, R., Turner, L.M., Smoski, M.J., Pozdol, S.L., \& Stone, W.L. (2003). Visual Recognition of Biological Motion is Impaired in Children with Autism. Psychological Science, 14, 151-157.

Cermak, S.A., Coster, W., \& Drake, C. (1980). Representational and non-representational gestures in boys with learning disabilities. American Journal of Occupational Therapy, 34, 19-26.

Cody, H., Pelphrey, K., \& Piven, J. (2002). Structural and functional magnetic resonance imaging of autism. International Journal of Developmental Neuroscience, 20, 421438.

De Meyer, M.K., Alpern, G.D., Barton, S., De Meyer, W.E., Churchill, D.W., Hingtgen, J.N., Bryson, C.Q., Pontius, W., \& Kimberlin, C. (1972). Imitation in autistics, early schizophrenic, and nonpsychotic subnormal children. Journal of Autism and Childhood Schizophrenia, 2, 264-287.

Dunn, L.M., Thériault-Whalen, C.M., \& Dunn, L.M. (1993). Echelle de Vocabulaire en Images Peabody (EVIP). Circle Pines: American Guidance Services.

Erin, J.N. (1990). Language samples from visually impaired four and five-year olds. Journal of Childhood Communication Disorders, 13, 181-191.

Escalona, A., Field, T., Nadel, J., \& Lundy, B. (2002). Brief Report: Imitation Effects on Children with Autism. Journal of Autism and Developmental Disorders, 32, 141-144.

Field, T., Field, T., Sanders, C., \& Nadel, J. (2001). Children with Autism Display more Social Behaviors after Repeated Imitation Sessions. Autism, 5, 317-323.

Fox, R., \& Oross, S. (1990). Mental Retardation and Perception of Global Motion. Perception and Psychophysics, 48, 252-258.

Frith, U. (1989). Autism: explaining the enigma. Oxford, UK: Basic Blackwell.

Gepner, B. (2001). Malvoyance du mouvement dans l'autisme infantile? Une nouvelle approche neuropsychopathologique développementale. Psychiatrie de l'enfant, 1, 77126.

Gepner, B. (2006). Constellation autistique, mouvement, temps et pensée. Malvoyance de l'ÉMotion, autres désordres du traitement temporospatial des flux sensoriels et dyssynchronie dans l'autisme. Devenir, 18, 4, 333-379.

Gepner, B. \& Mestre, D. (2002a). Brief Report: Postural reactivity to fast visual motion differentiates autistic from children with Asperger syndrome. Journal of Autism and Developmental Disorders, 32, 231-238. 
Gepner, B., \& Mestre, D. (2002b). Rapid visual-motion integration deficit in autism. Trends in Cognitive Sciences, 8, 455.

Gepner, B., \& Tardif, C. (2006). Autism, movement, time and thought. E-motion mis-sight and other temporo-spatial processing disorders in autism. In M. Vanchevsky (Ed.), Frontiers in Cognitive Psychology (pp.71-101). New York: Nova Sciences Publishers.

Gepner, B., de Gelder, B., \& de Schonen, S. (1996). Face processing in autistics: a generalised deficit? Child Neuropsychology, 2, 123-139.

Gepner, B., Deruelle, C., \& Grynfeltt, S. (2001). Motion and emotion: a novel approach to the study of face processing by young autistic children. Journal of Autism and Developmental Disorders, 31, 37-45.

Gepner, B., Lainé, F., Tardif, C. (2005). E-Motion mis-sight and other temporal processing disorders in autism. Cahiers de Psychologie Cognitive/Current Psychology of Cognition, 23, 104-121.

Gepner, B., Mestre, D., Masson, G., \& de Schonen, S. (1995). Postural effects of motion vision in young autistic children. NeuroReport, 6, 1211-1214.

Goldenberg, G. (1996). Defective imitation of gestures in patients with damage in the left or right hemisphere. Journal of Neurology, Neurosurgery and Psychiatry, 61, 176-180.

Goldenberg, G. (1999). Matching and imitation of hand and finger postures in patients with damage in the left or right hemispheres. Neuropsychologia, 37, 559-566.

Grandin, T. (1997). Penser en images. Paris : Odile Jacob.

Green, D., Baird, G., Barnett, A.L., Henderson, L., Huber, H., \& Henderson, S.E. (2002). The severity and nature of motor impairment in Asperger's syndrome: a comparison with Specific Developmental Disorder of Motor Function. Journal of Child Psychology and Psychiatry, 43, 655-668.

Greenspan, S.I., \& Porges, S.W. (1984). Psychopathology in infancy and early childhood: Clinical perspectives on the organization of sensory and affective-thematic experience. Child Development, 55, 49-70.

Hansen, P.C., Stein, J.F., Orde, S.R., Winter, J.L., \& Talcott, J.B. (2001). Are dyslexics' visual deficits limited to measures of dorsal stream function? Neuroreport, 13, 843847.

Heimann, M., \& Ullstadius, E. (1996). Imitation et troubles développementaux. Enfance, 1, 35-37.

Hobson, P., Ouston, J., \& Lee, A. (1988). What's in a face ? The case of autism. British Journal of Psychology, 79, 441-453.

Kanner, L. (1943). Autistic disturbances of affective contact. Nervous Child, 2, 217-250.

Kaufman, A., \& Kaufman, N.L. (1993). Batterie pour l'examen psychologique de l'enfant ( $K$ $A B C)$. Paris : Edition du Centre de Psychologie Appliquée.

Kogan, C.S., Boutet, I., Cornish, K., Zangenehpour, S., Mullen, K.T., \& Holder, J.J.A. (2004). Differential impact of the FMR1 gene on visual processing in fragile $X$ syndrome. Brain, 127, 591-601.

Klin, A., Jones, W., Schultz, R., \& Volkmar, F. (2003). The enactive mind, or from actions to cognition: lessons from autism. Philosophical Transactions of the Royal Society of London B, 358, 345-360.

Lainé, F., Tardif, C., \& Gepner, B. (sous presse). Amélioration de la reconnaissance et de l'imitation d'expressions faciales chez des enfants autistes grâce à une présentation visuelle et sonore ralentie. Annales Médico-Psychologiques. DOI : 10.1016/J.AMP.2005.09.032.

Landry, R., \& Bryson, S.E. (2004). Impaired Disengagement of Attention in Young Children With Autism. Journal of Child Psychology and Psychiatry, 45, 1115-1122. 
Milne, E., Swettenham, J., \& Campbell, R. (2005). Motion perception and autistic spectrum disorders: A review. Cahiers de Psychologie Cognitive/Current Psychology of Cognition, 23(1), 3-36.

Milne, E., Swettenham, J., Hansen, P., Campbell, R., Jeffries, H., \& Plaisted, K. (2002). High motion coherence thresholds in children with autism. Journal of Child Psychology and Psychiatry, 43, 255-263.

Mottron, L., \& Belleville, S. (1998). L'hypothèse perceptive visuelle dans l'autisme. Psychologie Française, 43, 135-145.

Mottron, L., \& Belleville, S. (2003). Locally oriented perception with intact global processing among adolescents with high functionning autism: Evidence from multiple paradigms. Journal of Child Psychology and Psychiatry, 6, 906-913.

Nadel, J. (2004). Do children with autism understand imitation as intentional interaction. Journal of Psychology and Behavioral Psychotherapy, 4, 165-177.

Nadel, J. (2005). Imitation et Autisme. In A. Berthoz et al. (Eds.), Autisme, Cerveau et Développement (pp.341-356). Paris. Odile Jacob.

Nadel, J. (2006). Does Imitation Matter to Children with Autism? In S.J. Rogers \& J.H.G. Williams (Eds.), Imitation and the Social Mind. Autism and Typical Development (pp. 118-137). New York: Guilford Publications.

Nadel, J., \& Aouka, N. (2006). Imitation: Some Cues for Intervention Approaches in Autism Spectrum Disorders. In T. Charman, \& W. Stone (Eds.), Social and Communication Development in Autism Spectrum Disorders: Early Identification, Diagnosis and Intervention.

Nadel, J., \& Potier, C. (2002). Imiter et être imité dans le développement de l'intentionnalité. In J. Nadel, \& J. Decety (Eds.), Imiter pour découvrir l'humain: Psychologie, neuropsychologie, robotique et philosophie de l'esprit (pp. 83-104). Paris : PUF.

Nadel, J., Croue, S., Kervella, C., Mattlinger, M.J., Canet, P., Hudelot, C., Lecuyer, C., \& Martini, M. (2000). Do autistic children have ontological expectancies concerning human behavior? Autism, 4, 133-145.

Rogers, S.J., \& Pennington, B.F. (1991). A theoretical approach to the deficits in infantile autism. Developmental Psychopathology, 3, 137-162.

Russell, J. (1997). Autism as an executive disorder. Oxford: Oxford University Press.

Schopler, E., \& Reichler, R.J. (1979). Individualized assessment and treatment for autistic and developmentally disabled children. Vol. 1: Psychoeducational profile (2ème Ed). Austin, TX, Pro-Ed, trad. Fr. Profil Psycho-éducatif révisé (PEP-R), Bruxelles, De Boeck Université, 1994.

Schopler, E., Reichler, R..J, de Vellis, R.F., \& Daly, K. (1980). Toward objective classification of childhood autism: Childhood Autism Rating Scale (CARS). Journal of Autism and Developmental Disorders , 10, 91-103.

Smith, I.M., \& Bryson, S.E. (1994). Imitation and Action in Autism: A Critical Review. Psychological Bulletin, 116, 259-273.

Sparrow, W.A., Shinkfield, A.J., Day, R.M., \& Zerman, L. (1999). Visual perception of human activity and gender in biological motion displays by individuals with Mental Retardation. American Journal of Mental Retardation, 104,215-226.

Spencer, J., O’Brien, J., Riggs, K., Braddick, O., Atkinson, J., \& Wattam-Bell, J. (2000). Motion processing in autism; evidence for a dorsal stream deficiency. NeuroReport, 11, 2765-2767.

Takarae, Y., Minshew, N.J., Luna, B., Krisky, C.M., \& Sweeney, J.A. (2004). Pursuit eye movement deficits in autism. Brain, 127, 2584. 
Talcott, J.B., Hansen, P.C., Assoku, E.L., Stein, J.F. (2000). Visual motion sensitivity in dyslexia: evidence for temporal and energy integration deficits. Neuropsychologia, 38, 935-943.

Tardif, C., \& Plumet, M.H. (2000). Détection des répertoires d'interaction sociale propres à chaque enfant autiste : enjeux pour la recherche et la clinique. In C. Riboni \& V.Gérardin-Collet (Eds), Autisme : perspectives actuelles (pp.45-60). Paris: L'Harmattan.

Tardif, C., Lainé, F., Rodriguez, M., \& Gepner, B. (2007). Slowing down presentation of facial movements and vocal sounds enhances facial expression recognition and induces facial-vocal imitation in children with autism. Journal of Autism and Developmental Disorders, 37, 1469-1484.

Williams, J.H.G., Whiten, A., \& Singh, T. (2004). A Systematic Review of Action Imitation in Autistic Spectrum Disorder. Journal of Autism and Developmental Disorders, 34, 285-299.

Williams, D.L., Goldstein, G., \& Minshew, N.J. (2006). Neuropsychologic functioning in children with autism: further evidence for disordered complex information-processing. Child Neuropsychology, 12, 279-298.

World Health Organisation (Organisation Mondiale de la Santé) (1994). International Classification of Disease (ICD-10). Genève: World Health Organisation.

\section{ANNEXE}

Description des gestes à imiter :

1. Sauter deux fois

2. Faire le «moulin» (passer les bras alternativement l'un au-dessus de l'autre devant le buste)

3. Lever les bras au-dessus de la tête

4. Taper les mains sur les cuisses en alternance

5. Une main derrière la tête, le coude sur le côté du corps, l'autre main vient toucher le coude en passant le bras par le bas

6. Fermer les yeux

7. Gonfler les joues

8. Tirer la langue

9. Ouvrir la bouche modérément

10. La main fermée le pouce dehors passe du haut vers le bas

11. La main ouverte, paume vers la caméra, vient se fermer, le pouce sur les autres doigts

12. Les bras devant le buste et les mains dans la continuité des bras, parallèles au sol forment des cercles dans l'espace simultanément et en symétrie

13. La main ouverte paume vers la caméra, les doigts tendus et écartés les uns des autres, le pouce vient toucher successivement tous les autres doigts en commençant par l'index et en terminant par l'auriculaire pour venir finalement se replacer dans sa position initiale

14. La main ouverte paume vers le plafond, les doigts se rejoignent tous entre eux pour former une sorte de tunnel

15. La main ouverte paume vers le sol, les doigts tendus resserrés les uns contre les autres, la main se retourne pour finir la paume vers le ciel

16. Les bras le long du cors viennent s'ouvrir face la caméra comme pour prendre quelqu'un dans ses bras 
17. L'index pointé vers la caméra, le bras replié sur le buste vient s'étirer en direction de la caméra.

18. La main le long du corps vient toucher le haut de la tête

19. L'index pointé devant la caméra, au niveau du visage, vient toucher le nez

20. Les bras repliés sur le buste de profil à la caméra, s'étirent parallèles au sol pour finir tendus, les mains perpendiculaires aux bras

Sources de ces gestes :

Les gestes 1, 2, 7 et 8 sont issus de l'étude de Beadle-Brown et Whiten (2004).

Les gestes 4, 5, 6, 9, 10,11, 15, 17, 18, 19 et 20 sont issus et inspirés d'items du K-ABC de Kaufman et Kaufman (1993).

Les gestes 3, 13 et 14 sont issus et inspirés du PEP-R de Schopler et Reichler (1979).

Les gestes 12 et 16 sont issus du Gesture Test de Cermak, Coster et Drake (1980). 